\title{
Development of low-intensity home-based resistance training combined with physical activity counseling for elderly patients with advanced cancer : the exercise component of the NEXTAC program
}

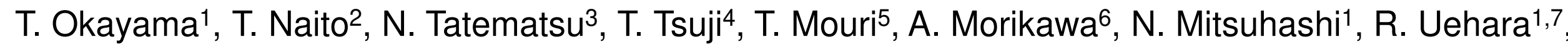

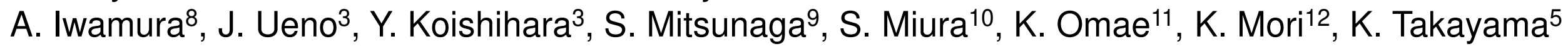

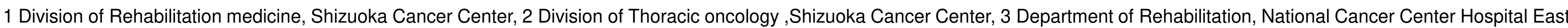
4 Department of Rehabilitation medicine, Keio University School of Medicine, 5 Department of Pulmonary Medicine ,Kyoto Prefectural University of Medicine

6 Department of Nursing ,Shizuoka Cancer Center, 7 Division of Rehabilitation medicine ,Kanagawa Cancer Center, 8 Department of Rehabilitation, Niigata Cancer Center Hospital

9 Department of Hepatobiliary Pancreatic Oncology ,National Cancer Center Hospital East , 10 Department of Internal Medicine, Niigata Cancer Center Hospital

11 Department of Clinical Biostatistics ,Kyoto University, 12 Clinical Research Center ,Shizuoka Cancer Center

\section{Intro duc tion}

The NEXTAC ( Nutrition and Exercise Treatment for Advanced Cancer ) program was a multimodal intervention for elderly patients with advanced cancer to maintain or improve their nutritional status and physical function during systemic chemotherapy.

\section{Aims}

The aim of this presentation is to show algorithm of the exercise prescription , compliance and safety

【Study design】

\section{Me thod}

This is the multi-center prospective single-arm study.

【Patient selection】

(Clinical Trial Registry No. UMIN000023207)

1) Pathological proven advanced non-small-cell lung cancer or pancreatic cancer

2) $\geq 70$ years old and ECOG-PS 0-2

3) They planned to receive the first-line systemic chemotherapy

4) Not having high risks for safe physical assessment

5) The ability to ambulate, read, and respond to questions without assistance.

【Assessments and intervention】

informed consent $\quad \mathrm{T} 1$

$\mathrm{T} 2$

T3

(screening period)

0

$4 \pm 2$ week

$8 \pm 2$ week

Table 1:Patient characteristics

\begin{tabular}{|l|c|}
\hline \multicolumn{1}{|c|}{ Variables } & $\mathrm{N}=30$ \\
\hline Age, median (range) & $75(70-84)$ \\
\hline Gender (Women:Men) & $10: 20$ \\
\hline ECOG-PS, n (\%) & $11(36.7)$ \\
0 & $19(63.3)$ \\
1 & \\
\hline Cancer type, n (\%) & $24(80.0)$ \\
Non-small-cell lung cancer & $6(20.0)$ \\
Pancreatic cancer & $3(10.0)$ \\
\hline Stage, n (\%) & $27(90.0)$ \\
III & \\
IV or postoperative recurrence & $20(66.7)$ \\
\hline Treatment, n (\%) & $10(33.3)$ \\
Cytotoxic regimen & $13(43.3)$ \\
Targeted regimen & $9(30.0)$ \\
\hline Comorbidities, n (\%) & $7(23.3)$ \\
Chronic lung disease & $3(10.0)$ \\
Type 2 diabetes & \\
Cardiovascular disease & \\
Double cancer & \\
\hline
\end{tabular}

\section{Home-based muscle training}

$>$ At T1,Patients performed each exercise 10-times repetition for 3 sets.

$>$ Physiotherapist chose optimal prescription according to the modified Borgscale.

\begin{tabular}{|c|c|c|}
\hline Level & Average steps & Target steps \\
\hline 1 & $0 \sim 2000$ & $2000+$ go outside \\
\hline 2 & $2001 \sim 2500$ & 4000 \\
\hline 3 & $2501 \sim 3500$ & 5000 \\
\hline 4 & $3501 \sim 4500$ & 6000 \\
\hline 5 & $4501 \sim 5500$ & 7000 \\
\hline 6 & $5501 \sim$ & 8000 \\
\hline
\end{tabular}

$>$ Instructors encouraged patients to increase their physical activity both indoors and outdoors referring to interviews.

Re sult

Table3:Compliance and adherence

\begin{tabular}{|l|c|c|c|}
\hline \multicolumn{1}{|c|}{ Period } & $\begin{array}{c}\text { Baseline to } \\
\text { T2 point }\end{array}$ & $\begin{array}{c}\text { T2 to T3 } \\
\text { point }\end{array}$ & Total \\
\hline Daily resistance training & $94(67-100)$ & $92(22-100)$ & $94(51-98)$ \\
\hline $\begin{array}{c}\text { Exercise diary fill-in day }(\%)^{1} \\
\text { Performance day }(\%)^{2}\end{array}$ & $56(4-79)$ & $17(0-88)$ & $41(3-79)$ \\
\hline Full program & $29(7-58)$ & $42(0-81)$ & $42(7-66)$ \\
\hline Self-modified program & $100(80-100)$ & $\begin{array}{c}100(83- \\
100)\end{array}$ & $98(85-100)$ \\
\hline $\begin{array}{c}\text { Physical activity } \\
\text { Accelerometer wear day } \\
(\geq 5 \mathrm{~h} / \text { day, \% })^{1}\end{array}$ & &
\end{tabular}

1: Median (interquartile range) proportion of days in which patients filled in exercise diaries, or wore accelerometer $\geq 5$ hours a day during each term.

2: Median (interquartile range) proportion of days in which patients performed full, modified of prescribed resistance training program during each term

Table4:Adverse events

\begin{tabular}{|l|}
\hline CTCAE v4.0Term \\
\hline Muscle pain \\
\hline Arthralgia \\
\hline Dyspnea \\
\hline Plantar aponeurosis \\
\hline
\end{tabular}

Grade
Grade 1
Grade 1
Grade 1
Grade 1

\section{Patients(n)}

2

1
$>$ Patients performed each exercise 10-times repetition for 3 sets a day at home.

Fig : 1 Home-based muscle training

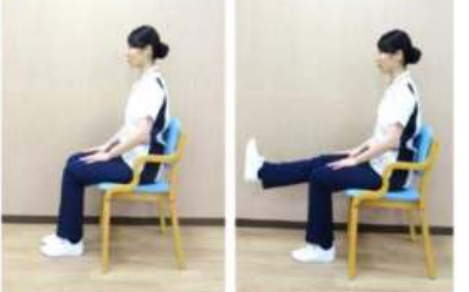

(1)Knee-extension

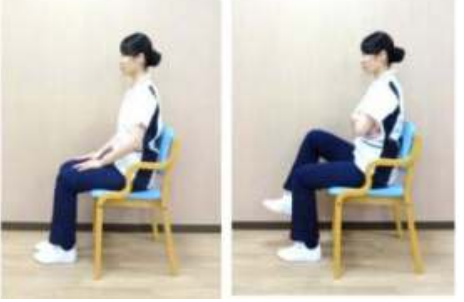

(2) Knee-raise

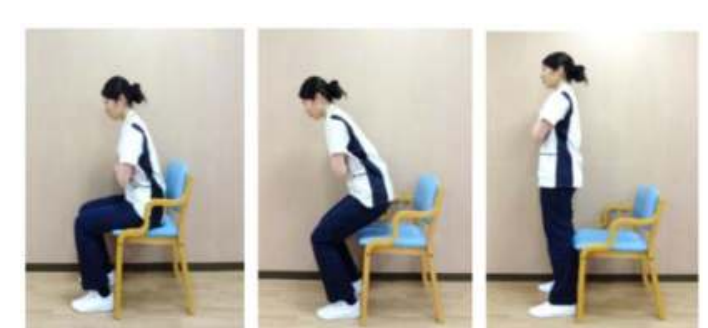

(3) Sit-to-Stand

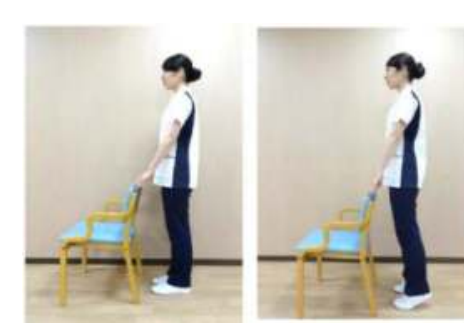

(4)Calf-raise

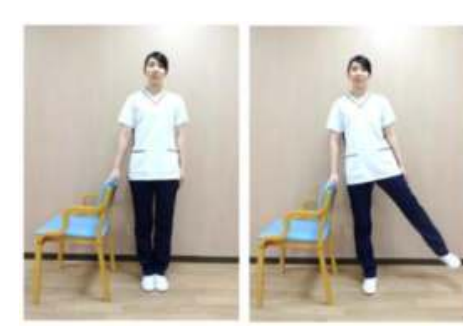

(5)Side-leg-raise
Table5:Changes in outcome measures

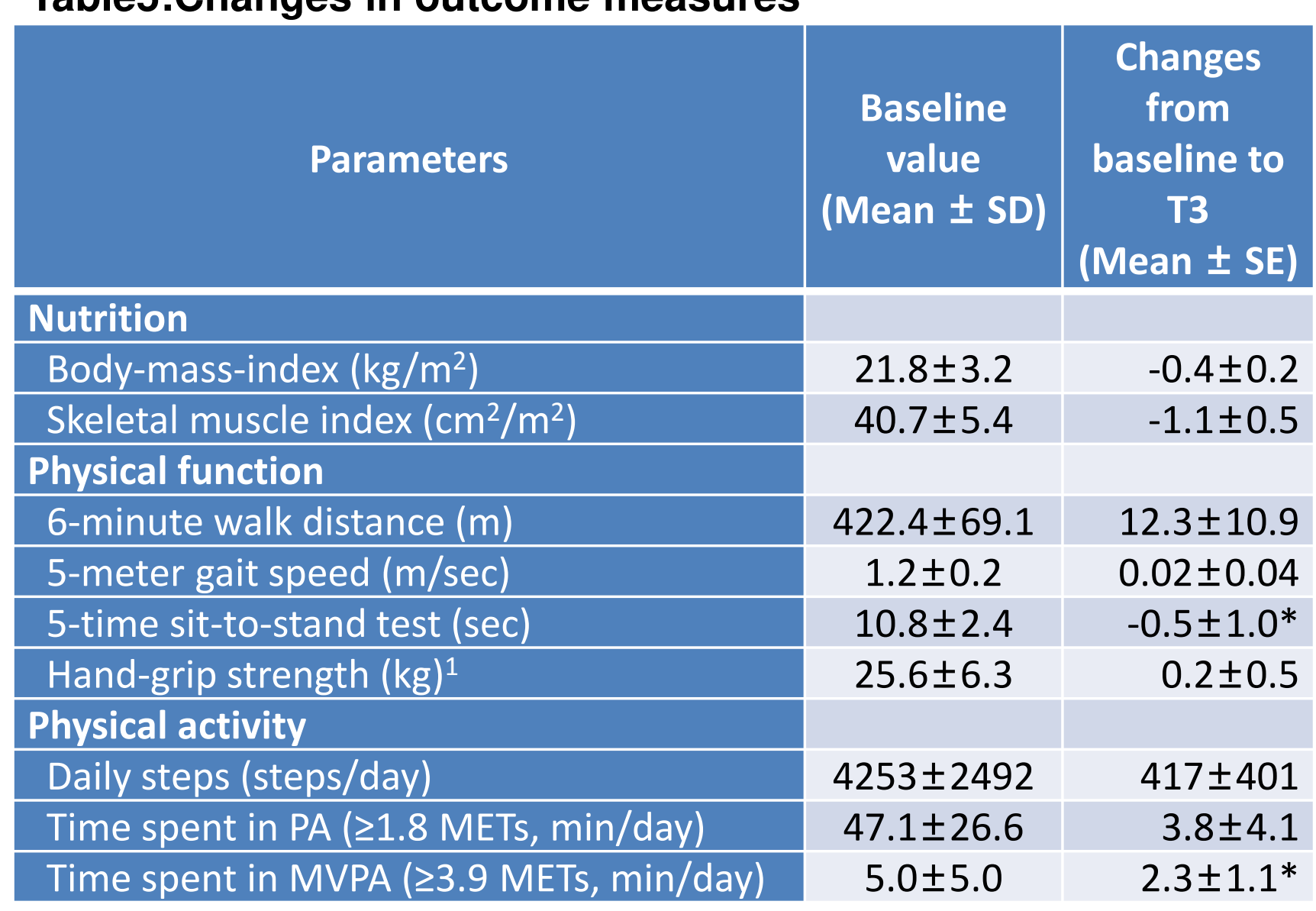

${ }^{*} \mathrm{P}<0.05$ in Wilcoxon signed-rank test

Conclusion

E Our exercise program was feasible and safe for the elderly patients with advanced cancer.

- We are now conducting a randomized phase two study to measure the impact of these interventions on functional prognosis. 